\begin{tabular}{|c|l|}
\hline Title & $\begin{array}{l}\text { Electron Transfer through Organic Monol ay ers Directly Bonded to Silicon Probed by Current Sensing A tomic Force } \\
\text { Microscopy : Effect of Chain Length and A pplied Force }\end{array}$ \\
\hline Author(s) & Zhao, Jianwei; Uosaki, Kohei \\
\hline Citation & $\begin{array}{l}\text { Journal of Physical Chemistry B, 108(44), 17129-17135 } \\
\text { https:/doi.org/10.1021/jp049719+ }\end{array}$ \\
\hline Issue Date & 2004_11-04 \\
\hline Doc URL & http://hdl.handle.net/2115/50226 \\
\hline Type & article \\
\hline File Information & JPCB108_44_17129-17135.pdf \\
\hline
\end{tabular}

Instructions for use 


\title{
Electron Transfer through Organic Monolayers Directly Bonded to Silicon Probed by Current Sensing Atomic Force Microscopy: Effect of Chain Length and Applied Force
}

\author{
Jianwei Zhao ${ }^{\dagger}$ and Kohei Uosaki* \\ Physical Chemistry Laboratory, Division of Chemistry, Graduate School of Science, Hokkaido University, \\ Sapporo, Japan, 060-0810
}

Received: January 20, 2004; In Final Form: September 3, 2004

\begin{abstract}
Electron transfer through organic monolayers directly bonded to a silicon surface has been investigated by using a current sensing atomic force microscope (CSAFM). The research system employs a platinum-coated CSAFM tip in point contact at a confined nanometer size with a set of alkyl monolayers of various chain lengths, which are covalently bonded directly to an $\mathrm{n}$-type $\mathrm{Si}(111)$ by immersing a H-terminated silicon substrate in neat $\mathrm{CH}_{2}=\mathrm{CH}-\left(\mathrm{CH}_{2}\right)_{n-3} \mathrm{CH}_{3}(n=12,14,16$, and 18) under heating. The current flows were analyzed with a modified electron-tunneling model and showed strong force and chain length dependences. Increasing the applied force resulted in a negative shift of the current-voltage $(I-V)$ curves. This observation was mainly explained by the amplified contact area and shortened tunneling distance. In addition, the $I-V$ curves showed chain length dependence, from which the bias-dependent electron tunneling coefficient, $\beta$, was analyzed.
\end{abstract}

\section{Introduction}

The attachment of organic monolayers to a silicon surface ${ }^{1}$ provides a direct combination of molecular materials and rigid semiconductor, making it a promising key technique in future silicon-based nanotechnology such as the development of highly integrated chemical and biological sensors ${ }^{2,3}$ and molecular electronic devices. ${ }^{4-8}$ Systematic study on the electric properties of the organic monolayers on the semiconductor substrate should also extend our understanding of surface electrochemistry, semiconductor electrochemistry, and electron transfer through organic/inorganic heterojunctions.

It has been proved that both the electron-transfer mechanism and electron-transfer rate strongly depend on the nature of the interfaces between organic molecules and solid substrates. ${ }^{9-13}$ Recently, some groups have proved that a chemical bond linking the organic molecule and electrode ${ }^{9-11}$ offers reliable electric contact, which shows reproducible electric behavior that is very close to theoretical prediction. ${ }^{11}$ Since Chidsey and co-workers $^{14,15}$ demonstrated the formation of alkyl monolayers when H-terminated $\mathrm{Si}(111)$ surfaces reacted with alkenes, that is, the generation of a chemical bond between silicon atoms and an alkyl monolayer, many methods such as thermal, ${ }^{15-19}$ photochemical, ${ }^{20-22}$ and electrochemical ${ }^{23,24}$ reactions and using a reaction with a radical initiator ${ }^{8,15,25,26}$ have been developed to directly bond organic molecules to a H-terminated $\mathrm{Si}(111)$ surface via $\mathrm{Si}-\mathrm{C}$ bonds. The electric properties of alkyl monolayers on silicon have been investigated utilizing silicon/ monolayer/electrolyte ${ }^{8,18,21}$ and silicon/monolayer/mercury drop junctions, ${ }^{27-29}$ but there is no general method to form the chemical bond between the organic molecule and a wide variety of metals. An alternative approach is to maintain physical contact between a metal tip and an organic monolayer with a constant stress. This can be achieved by using a conducting

* To whom correspondence should be addressed. E-mail: uosaki@ pcl.sci.hokudai.ac.jp.

†resent address: Department of Chemistry, Nanjing University, P. O. Box 240, Nanjing, 210093, P. R. China.

10.1021/jp049719+ CCC: \$27.50 atomic force microscope, which can exert stress on the monolayers through a conducting tip and measure the electric properties in a nanosized region. ${ }^{10-13,30,31}$ The reliability of this type of electric contact has been proved by several groups using metal-monolayer-metal junctions. Frisbie et al. studied electron transfer through a series of alkanethiol monolayers on gold and obtained an attenuation factor, $\beta$, of 1.45 per methylene. ${ }^{32}$ In their recent study, they applied this approach to electron transfer through the physically touched alkyl bilayers and obtained a similar attenuation factor of $1.1 \AA^{-1} .{ }^{12}$ Lindsay et al. studied the force and bias effects on the electron transfer for the same system but in a liquid and obtained $\beta$ values of $0.8 \pm$ 0.2 per methylene over a $\pm 3 \mathrm{~V}$ range. ${ }^{10}$ The contact resistance was also quantitatively characterized by Frisbie, showing 15$20 \mathrm{k} \Omega$ for a contact between an Au-coated conducting tip and an alkyl monolayer. ${ }^{13}$ This value is much smaller than that of the alkyl monolayers themselves. ${ }^{10,12,31,32}$

The study of electric properties of alkyl monolayers formed on hydrogen-terminated $\mathrm{Si}(111)$ has a very important advantage over previous studies on electric properties of alkyl monolayers on $\mathrm{SiOx} / \mathrm{Si}^{5,9}$ While a well-structured alkyl monolayer can be formed on a silicon substrate with a very clear interface of $\mathrm{Si}-\mathrm{C}$ bonds, the dimension and composition of the silicon oxide grown on silicon are difficult to control accurately.

Detailed studies on the formation kinetics and structure of alkyl monolayers on silicon by attenuated total reflection Fourier transform infrared spectroscopy (ATR-FTIR) and sum frequency generation (SFG) spectroscopy have shown that the monolayer was not only in the all-trans conformation, that is, in a high conformational order, but also in an epitaxial arrangement with the $\mathrm{Si}(111)$ substrate, that is, in a high lateral order. ${ }^{33,34}$

Here, we studied the electric properties of alkyl monolayers of various chain lengths on a $\mathrm{Si}(111)$ surface using a current sensing atomic force microscope (CSAFM) as shown in Scheme 1. Alkyl monolayers with different chain lengths were formed directly on the H-terminated $\mathrm{Si}(111)$ surface, and a Pt-coated AFM tip was brought into point contact with the monolayers 
SCHEME 1. Schematic Diagram of the Present System ${ }^{a}$

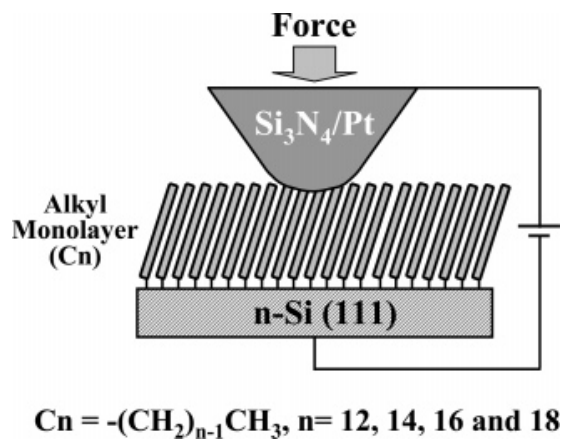

${ }^{a} \mathrm{~A}$ Pt-coated conducting AFM tip is brought into contact with alkyl monolayers of various chain lengths directly bonded to n-type $\mathrm{Si}(111)$.

at a certain stress. The effects of chain length and force on the electric properties of the research system were investigated in detail.

\section{Experimental Section}

Preparation of Alkyl Monolayers on Silicon. An alkyl monolayer coated $\mathrm{n}-\mathrm{Si}(111)$ was prepared according to the following procedure. Briefly, $n$-type $\mathrm{Si}(111)$ wafers $(0.002-$ $0.005 \Omega \mathrm{cm}$, P-doped) were cleaned in $3: 1 \mathrm{H}_{2} \mathrm{SO}_{4}$ (concentrated)/ $\mathrm{H}_{2} \mathrm{O}_{2}(30 \%)$ at $90{ }^{\circ} \mathrm{C}$ for $20 \mathrm{~min}$, followed by copious rinsing with Milli-Q water. The surfaces were etched with $5 \% \mathrm{HF}$ solution for $5 \mathrm{~min}$ and then reoxidized in $4: 1: 1 \mathrm{H}_{2} \mathrm{O} / \mathrm{HCl}$ (concentrated) $/ \mathrm{H}_{2} \mathrm{O}_{2}(30 \%)$ solution by boiling for $20 \mathrm{~min}$. Finally, the oxidized silicon wafers were etched with semiconductor grade $40 \%$ aqueous deoxygenated $\mathrm{NH}_{4} \mathrm{~F}$ for $10 \mathrm{~min}$ to obtain an atomically flat $\mathrm{H}$-terminated $\mathrm{Si}(111)$ surface. The silicon sample was quickly dried by blowing super pure argon and was then transferred to a reaction vessel, which contained the desired alkene reagent. The alkenes were of the highest purity available from Wako Chemicals and were used immediately after opening a new sample bottle without additional treatment, except for the $\mathrm{C} 16$, which was distilled at low pressure before use. The reaction was carried out at $200{ }^{\circ} \mathrm{C}$ for ca. $3 \mathrm{~h}$. Previous study on the monolayer formation showed that well-ordered alkyl monolayers were formed within $2 \mathrm{~h}$ under the same conditions. ${ }^{33,34}$ After cooling, the modified silicon samples were sequentially rinsed with diethyl ether, ethanol, and dichloromethane and then kept in $\mathrm{CH}_{2} \mathrm{Cl}_{2}$.

Ellipsometric Measurements. Ellipsometry measurements were carried out with a SOPRA GESP-5 spectrometer using a $30 \mathrm{~W}$ Xe lamp as a light source. The incident angle was set at $75^{\circ}$. The $\Psi$ and $\Delta$ values were measured between 300 and 800 $\mathrm{nm}$ with a step of $10 \mathrm{~nm}$. Simulations were carried out using the software provided by the manufacturer (SOPRA, WinElli) as follows. The complex dielectric function for the gold substrate, $\epsilon_{\mathrm{Au}}(v)$, was determined using a bare gold substrate. The dielectric functions for the alkyl monolayers were postulated to have no imaginary parts. A three-layer model (bulk silicon, alkyl film, and air) was used to evaluate the alkyl layer thickness from the ellipsometric data. The thickness was finally obtained by averaging the data acquired at three positions for each sample using three samples for each alkyl monolayer.

CSAFM Measurements. A CSAFM (Molecular Imaging) was used for both imaging and electric characterization. A Ptcoated $\mathrm{Si}_{3} \mathrm{~N}_{4}$ tip (MikroMasch; spring constant, $0.12 \mathrm{~N} / \mathrm{m}$; apex radius of curvature, $<35 \mathrm{~nm}$ ) was brought into contact with alkyl monolayers. Bias was applied to the substrate through an ohmic contact. Thus, the potential was given as the substrate potential with respect to the tip. To investigate the $I-V$ behavior of the monolayers, we stopped the CSAFM tip at a certain position on a flat silicon terrace and then made a bias scan, typically with $1-5 \mathrm{~V} / \mathrm{s}$, and recorded the current response. To record the second $I-V$ curve at a different position, the force was reduced as much as possible, and then the tip was moved to a new position at least $40 \mathrm{~nm}$ away, where a desired force load was applied and the bias scan was repeated. Twenty $I-V$ curves were recorded for each force, and the currents were then averaged. All electric measurements were carried out in air and under ambient conditions $\left(20 \pm 2{ }^{\circ} \mathrm{C}\right.$ and $30-40 \%$ humidity).

\section{Results and Discussion}

General Features of Alkyl Monolayers on Si(111). Since the reaction time was $3 \mathrm{~h}$ in the present experiments, the silicon substrate was expected to be fully covered with highly ordered alkyl monolayers. This was demonstrated by ellipsometry measurements, showing the thickness $\left(d_{n}\right)$ of $1.64 \pm 0.03,1.88$ $\pm 0.03,2.06 \pm 0.05$, and $2.32 \pm 0.03 \mathrm{~nm}$ for $\mathrm{C} 12, \mathrm{C} 14, \mathrm{C} 16$, and $\mathrm{C} 18$ monolayers, respectively. No obvious difference was found among the thicknesses for different positions and different samples as long as the same alkene reagent was used, implying that the alkyl monolayer formed by this method was homogeneous.

The morphologies of a $\mathrm{Si}(111)$ surface before and after organic monolayer modification were observed in the normal AFM mode, showing atomically flat terraces separated by a step height of ca. $0.3 \mathrm{~nm}$. Almost no pits or defects were observed. These features are very similar to those previously reported, although different methods were employed for the monolayer formation. ${ }^{16}$

The interaction between the Pt-coated CSAFM tip and the substrates was estimated by the attractive force measured from the force curves. The force curve for the contact AFM mode describes the deflection signal as a function of the displacement of the piezo position in the approaching and withdrawing operations. The deflection signal is proportional to the tip bending. The attractive force $(F)$ can be deduced by the deflection signal difference ( $\Delta D$ in volts) between the plateau and the bottom of the force curve according to $F=\Delta D(K / S)$, where $K$ is the spring constant of the tip $(\mathrm{N} / \mathrm{m})$ and $S$ is the sensitivity $(\mathrm{V} / \mathrm{nm})$ obtained from the slope of the relation between the deflection signal and the displacement of the piezo after tip-sample contact. The averaged adhesive force $\left(F_{0}\right)$ between the Pt-coated tip and the H-terminated silicon was 1.2 $\pm 0.3 \mathrm{nN}$, whereas the averaged attractive force between the Pt-coated tip and the $\mathrm{C} 12$ monolayer was $11.0 \pm 1.9 \mathrm{nN}$. Because the force measurements for the H-terminated and the monolayer-modified $\mathrm{Si}(111)$ surfaces were carried out under the same conditions, the difference in the attractive force should be caused only by the differences in the nature of the sample surfaces.

Breakdown Behavior of Alkyl Monolayers on Si(111). When bias is applied between the tip and substrate, the alkyl monolayer is subject to a very large electric field. For example, when the distance between the tip and substrate is around $2 \mathrm{~nm}$ and the bias of $-5 \mathrm{~V}$ is applied, the field is as high as $2.5 \times$ $10^{9} \mathrm{~V} / \mathrm{m}$. Therefore, breakdown of the alkyl monolayer is expected to occur when the bias becomes greater than a critical value. ${ }^{12,35,36}$ Breakdown of the monolayers was indeed observed but only when the applied force was relatively small. Typical breakdown $I-V$ curves for various chain lengths at a force less than $2 \mathrm{nN}$ are given in Figure 1, showing a sudden current jump from almost zero to over the measurement limit at a breakdown 


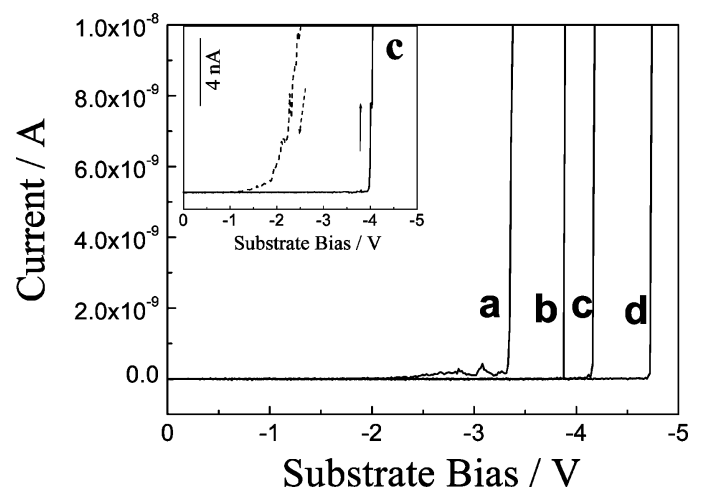

Figure 1. $I-V$ curves for (a) $\mathrm{C} 12$, (b) $\mathrm{C} 14$, (c) $\mathrm{C} 16$, and (d) $\mathrm{C} 18$ monolayers at a force less than $2 \mathrm{nN}$, showing breakdown behavior. Inset: $I-V$ relation for the $\mathrm{C} 16$ monolayer with bias scan.

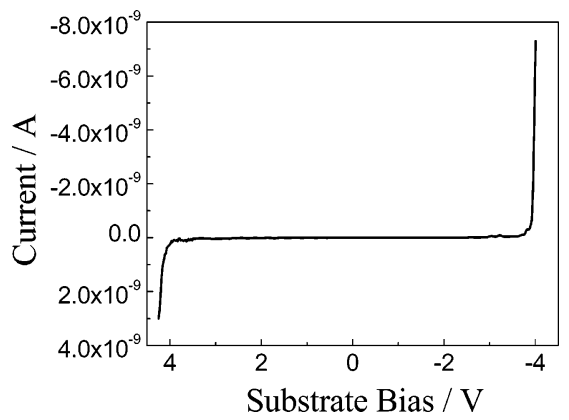

Figure 2. A Typical $I-V$ curve of $n-S i(111)$ modified with a $\mathrm{C} 12$ monolayer at $4.4 \mathrm{nN}$. The positive and negative branches of the $I-V$ curve were obtained by scanning the bias from zero in the positive and negative directions, respectively.

voltage. When the bias was scanned back, a current hysteresis was observed as shown in the inset of Figure 1 for the C16 monolayer.

The breakdown voltage was found to be dependent on the monolayer thickness as

$$
V_{\text {breakdown }}(\mathrm{V})=E \times \text { thickness }(\mathrm{nm})
$$

The dielectric strength, $E$, was determined to be $2.0 \mathrm{~V} / \mathrm{nm}$, the same as that reported for the alkyl monolayers on a gold substrate. ${ }^{12}$ This result also proved that the alkyl monolayer fabricated on $\mathrm{H}-\mathrm{Si}(111)$ is as closely packed and well ordered as those fabricated on a gold surface.

Chain Length Dependence of $\boldsymbol{I}-\boldsymbol{V}$ Behavior. A more reliable physical contact is expected with a greater applied force, leading to well-behaved $I-V$ curves for these junctions. A typical $I-V$ curve of the $\mathrm{C} 12$ monolayer on silicon at $4.4 \mathrm{nN}$ is shown in Figure 2.

Different behaviors were observed at positive and negative substrate biases. While the $I-V$ curve was stable even for dozens of bias scans as long as the bias was limited in the negative substrate bias region, it changed rapidly as soon as the bias was scanned in the positive substrate bias region. In the first positive bias scan, the current increased as it did in the negative bias region but the current decreased very rapidly and the first trace was no longer repeated at the second bias scan. Almost no current flowed for the third scan. Note that the $I-V$ curve given in Figure 2 corresponds to the first positive bias scan. This behavior was observed even when the measurements were carried out in a chamber filled with inert gas and has been attributed to the oxidation of silicon in the presence of residual water and oxygen in the environment. ${ }^{37,38} \mathrm{~A}$ similar phenomenon was also observed for $\mathrm{H}$-terminated silicon. The only difference

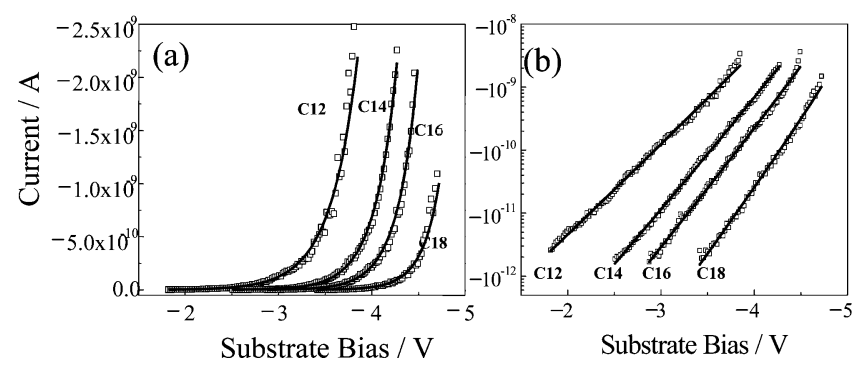

Figure 3. Chain length dependence of $I-V$ curves obtained at $5.9 \pm$ $0.3 \mathrm{nN}$ for alkyl monolayers on $\mathrm{n}-\mathrm{Si}(111)$. The current is given in (a) linear coordinates and (b) log coordinates. Squares represent experimental data, and solid curves are the simulated results using the modified electron tunneling model.

was that a much smaller substrate bias was required for the oxidation of H-terminated silicon, indicating that the alkyl monolayers passivate the silicon surface much more. ${ }^{7}$ To avoid substrate oxidation, the $I-V$ relation was measured only at negative substrate bias in this study.

The $I-V$ curves obtained at negative bias showed no obvious bias scan speed dependence. In the present experiments, when the bias scan speed was varied from 0.05 to $100 \mathrm{~V} / \mathrm{s}$, only a slight change was observed in the $I-V$ curves, indicating that electron transfer through the junctions was very fast.

Typical $I-V$ curves for all samples at a constant force of 5.9 $\pm 0.3 \mathrm{nN}$ are shown together in Figure $3 \mathrm{a}$. An increase in chain length resulted in a negative shift of the $I-V$ curves. Semilog plots of the $I-V$ curves are given in Figure 3b, which shows linear relations between $\log (i)$ and $V$. These results indicate that the longer the alkyl chain, the thicker the barrier for the electron transfer. ${ }^{39}$ In other words, a smaller current flows for a longer alkyl chain at a given bias. The chain length dependencies of $\log (i)$ at various biases are given in Figure $4 \mathrm{a}$. The values of $\log (i)$ linearly decrease with increase in the number of the carbon chain, that is, the chain length.

Electron transfer through alkyl monolayers follows the tunneling relation: ${ }^{10,12,31,32}$

$$
i=i_{0} \exp (-\beta d)
$$

where $d$ is the thickness of the barrier through which the electron transfer takes place, and $\beta$ is the structure-dependent attenuation factor, that is, tunneling coefficient, which can be obtained from the slopes of the $\log (i)$ and $V$ relations. $\beta$ was clearly dependent on bias as shown in Figure 4b. Obviously, higher bias led to smaller $\beta$. The bias-dependent $\beta$ can be interpreted by the Simmons model that considers electron tunneling through a rectangular barrier. ${ }^{40}$ The tunneling coefficient, $\beta$, can be expressed as

$$
\beta=\frac{4 \pi}{h} \sqrt{2 m(\phi-q V / 2)}
$$

where $m$ and $\phi$ denote electron mass and tunneling barrier height, respectively, with the other symbols having their usual meanings. This model predicts a bias dependence of $\beta$ as proved in this study. However, when the bias is much smaller than the barrier height, bias dependence is not obvious. This has been demonstrated by Lindsay et al. for electron transfer through a series of alkanethiol self-assembled monolayers (SAMs) on gold. ${ }^{10}$ In a wide bias range from -3 to $3 \mathrm{~V}$, they did not observe obvious bias dependence of $\beta .{ }^{10}$ When the bias is comparable to the barrier height, an obvious bias dependence of $\beta$ is as expected as shown in Figure $4 \mathrm{~b}$, where the squares represent $\beta$ values measured from the chain length dependence 

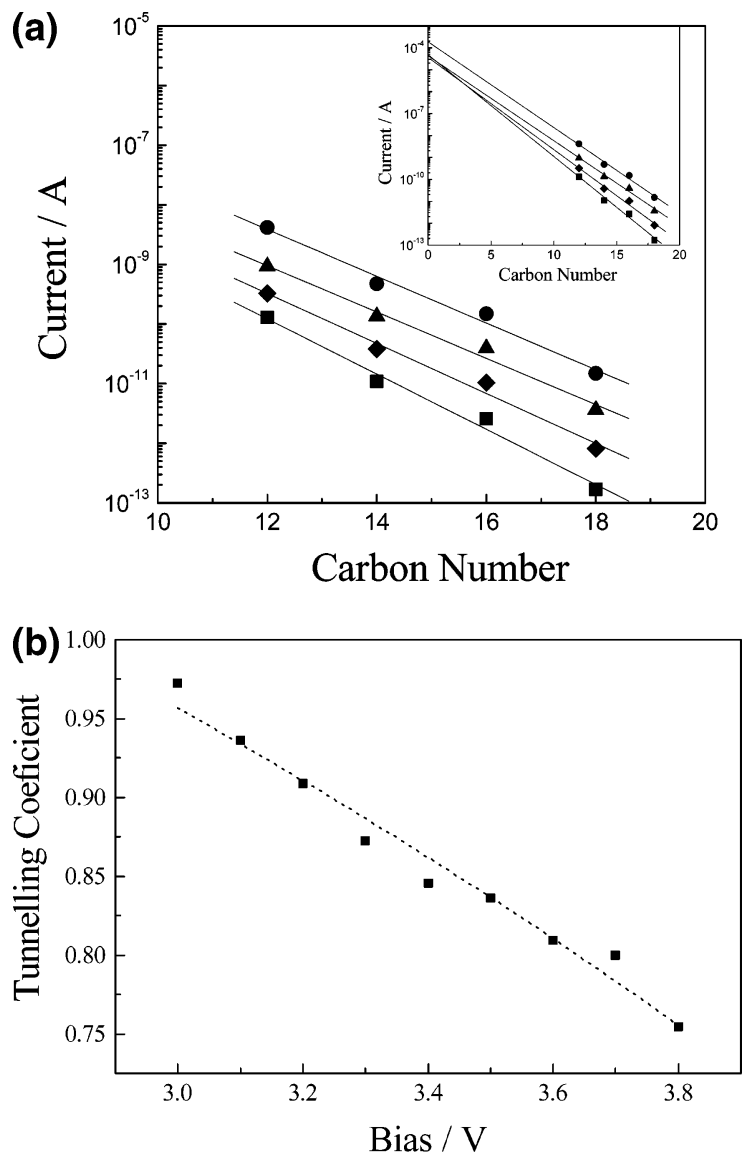

Figure 4. (a) Chain length dependence of the current at $-3.0 \mathrm{~V}$ (square), $-3.3 \mathrm{~V}$ (diamond), $-3.6 \mathrm{~V}$ (triangle), and $-3.8 \mathrm{~V}$ (circle). The current values at zero carbon number were obtained by extrapolation as shown in the inset. (b) Bias dependence of the tunneling coefficient. Each tunneling coefficient symbolized by a square was obtained from the slope of the chain length dependence of the current at a given bias as shown in panel a, and the dotted line shows the results of the theoretical simulation using eq 3 .

of current at a given bias. Assuming that most of the potential drop occurs within the alkyl monolayer as demonstrated later, the tunneling barrier height, $\varphi$, of $2.56 \pm 0.05 \mathrm{eV}$ was obtained by fitting the $\beta-V$ relation with eq 3 . The theoretically simulated results using eq 3 are shown by a dotted line in Figure $4 \mathrm{~b}$. In addition, $\beta$ at zero bias was found to be $1.48 \AA^{-1}$. These values are close to those reported previously..$^{10,12,31,32}$

One important question to be answered is why the breakdown takes place only at a force less than $2 \mathrm{nN}$, while well-behaved $I-V$ curves are observed at greater forces. It is worth considering the role of interfacial resistance of the physical contact between the organic monolayer and the conducting tip. Since only a small force of $5.9 \pm 0.3 \mathrm{nN}$ was applied, the deformation of monolayers was rather small. The interfacial resistances can be deduced by extrapolation to zero carbon number as shown in the inset of Figure $4 \mathrm{a}$. The interfacial resistance is $84 \pm 15$ $\mathrm{k} \Omega$, which is comparable to the quantum unit of resistance $R_{\mathrm{Q}}$ $\left(h / e^{2} \sim 26 \mathrm{k} \Omega\right)$. A smaller force may lead to a looser physical contact, that is, larger interfacial resistance. Generally, in a double-junction structure, if each junction resistance is much higher than the $R_{\mathrm{Q}}$ due to a long electron tunneling distance or a high tunneling barrier, the electron transport occurs in two independent sequential steps with possible occupation on the molecular tunnelling bridge. ${ }^{41}$ In our experiments, the resistance of the alkyl monolayers should be greater than $10^{10} \Omega$. In addition, the interfacial resistance of the physical contact at a force less than $2 \mathrm{nN}$ is also much higher than the $R_{\mathrm{Q}}$. Therefore, two-step sequential electron transfer via the organic monolayer and the physical contact was realized. In this case, the electrons may accumulate on the molecular bridge, leading to breakdown. However, when the interfacial resistance is much smaller than the $R_{\mathrm{Q}}$ at higher force, the physical contact becomes electrically reliable and can be treated as conductive.

A comparison of the electric behavior of the semiconductormonolayer-metal junction with that of metal-monolayermetal junctions is very instructive in determining the electrontransfer mechanism. For the latter, it is known that the electron transfer can be explained by a single-step electron transfer, that is, simple electron tunneling, mechanism. ${ }^{10,12,31,32}$ On the other hand, almost no current was detected in the small bias region for the semiconductor-monolayer-metal junctions (Figure $3 \mathrm{a}$ ), and an almost linear relation between $\log (i)$ and $V$ was observed at sufficiently high bias (Figure 3b). This implies that the simple electron tunneling model is not valid for semiconductormonolayer-metal junctions. A modified electron tunneling mechanism should be considered to describe the electron transfer through alkyl monolayers on silicon. This model is schematically illustrated in Figure 5a with the band structure shown in Figure 5b. Two basic steps are involved in this process. First, there is a thermal equilibrium for free electrons between the interface ( $\left.N_{\mathrm{e}, \text { surf }}\right)$ and the bulk $\left(N_{\mathrm{e}, \text { bulk }}\right)$ of silicon. ${ }^{42}$ The free electrons at the interface may tunnel through the alkyl monolayers to the metal electrode. The formula for current density based on this model is given by ${ }^{39}$

$$
i_{x}=A^{*} T^{2} \exp \left(\frac{-q \phi_{B 0}}{k T}\right) \exp \left(\frac{\alpha q V}{k T}\right) \exp (-\beta \delta)
$$

or in a logarithmic format as

$$
\ln \left(i_{x}\right)=\left[\ln \left(A^{*} T^{2}\right)-q \phi_{B 0} / k T\right]+\alpha q V / k T-\beta \delta
$$

where $A^{*}$ is the Richardson constant, $T$ is temperature, $k$ is the Boltzmann constant, $\phi_{B 0}$ is the Schottky barrier height, and $\delta$ ( $)$ is the thickness of the tunneling barrier, that is, the alkyl monolayer in this case. By replacing the tunneling coefficient, $\beta$, with eq 3 , the current density can be rewritten as

$$
\begin{aligned}
i_{x}=A^{*} T^{2} \exp \left(\frac{-q \phi_{B 0}}{k T}\right) \exp \left(\frac{\alpha q V}{k T}\right) \\
\quad \exp \left(-\delta \frac{4 \pi}{h} \sqrt{2 m[\phi-(1-\alpha) q V / 2]}\right)
\end{aligned}
$$

or

$$
\ln \left(i_{x}\right)=\left[\ln \left(A^{*} T^{2}\right)-\frac{q \phi_{B 0}}{k T}\right]+\frac{\alpha q V}{k T}-\frac{4 \pi(2 m)^{1 / 2}}{h}[\phi-(1-
$$

The factor $\alpha$, in eqs 4 and 5 , represents the fraction of the potential drop within the semiconductor side $\left(V_{\mathrm{s}}\right)$ over the whole applied bias $(V)$ and is defined as

$$
\alpha=V_{\mathrm{s}} / V
$$

Equations 4 and 5 are valid only for forward bias with $V>3$ $k T / q$, where reverse electron tunneling from the metal to the semiconductor can be neglected. Calculated results using eq 5 with an adjustable pre-exponential factor are also shown in Figure 3a,b. The simulated current curves in both linear and logarithmic coordinates are in good agreement with the experi- 


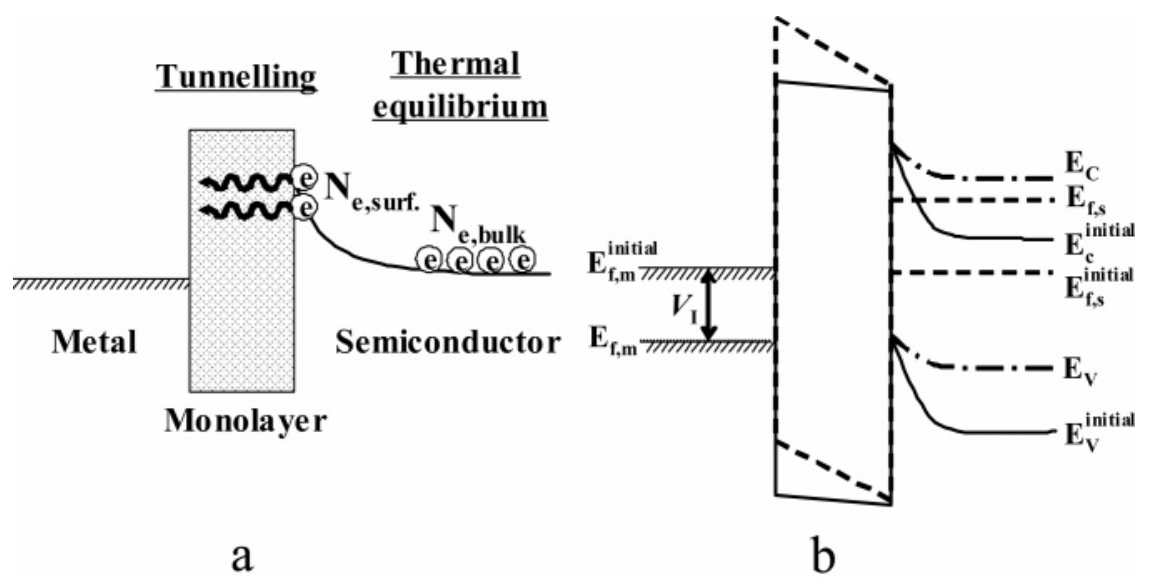

Figure 5. (a) Schematic diagram and (b) energy band structure for the modified electron tunneling model. $N_{\mathrm{e}, \text { surf }}$ and $N_{\mathrm{e}, \text { bulk }}$ are the number of electrons at the surface and in the bulk, respectively. $E_{\mathrm{f}, \mathrm{m}}$ and $E_{\mathrm{f}, \mathrm{s}}$ are the Fermi levels in the metal and semiconductor, respectively. $E_{\mathrm{c}}$ and $E_{\mathrm{v}}$ are the conduction band and valence band, respectively. The superscript initial corresponds to the situation at zero bias.

mental data points, implying that the model can well explain the electron-transfer behavior under the present experimental conditions.

By simulating the experimental data using the modified electron tunneling model, the $\alpha$ values, that is, the fraction of potential drop within the semiconductor, was obtained from the slope of the $\ln \left(i_{x}\right)$ and $V$ plot. The averaged $\alpha$ values that show only slight force dependence were $0.09 \pm 0.01,0.10 \pm 0.01$, $0.10 \pm 0.01$, and $0.10 \pm 0.01$ for $\mathrm{C} 12, \mathrm{C} 14, \mathrm{C} 16$, and $\mathrm{C} 18$ monolayers, respectively. All results indicated that most of the potential drop occurred within the insulator, that is, monolayer, and only about $10 \%$ of the potential drop occurred within the semiconductor. The $\alpha$ values in the present study were much smaller than those of inorganic metal-insulator-semiconductor (MIS) junctions: for example, $1 / 2.7-1 / 3.4$ for $\mathrm{Al} / \mathrm{SiOx} /$ p-Si(100) ${ }^{43}$ ca. 1/1.3 for Ni/Oxide/GaAs, ${ }^{44}$ and ca. $1 / 1.8$ for $\mathrm{Au} / \mathrm{SiOx} / \mathrm{n}-\mathrm{Si} .{ }^{39}$ Very high resistivities of the alkyl monolayers are the main reason for this observation.

Force-Dependent $\boldsymbol{I}-\boldsymbol{V}$ Relations. The force dependence of the $I-V$ curves was studied in detail using the same Pt-coated tip for a $\mathrm{C} 12$ monolayer with force loads ranging from 4.4 to $46.2 \mathrm{nN}$ as shown in Figure 6. A force higher than these values may lead to not only irreversible $I-V$ behaviors but also pattern formation on the surface, which should be the result of the collapse of the monolayer structure as we reported previously ${ }^{30}$ and actually can be considered as a basis for AFM-based nanolithography. ${ }^{45}$ To avoid the uncertainty in the measurement caused by tip scratching, the current signal was recorded as follows. After the tip had been brought into contact with the sample surface, the tip stress was increased to a certain value and then the current signal was recorded. To record the second $I-V$ curve at a different position, the force was reduced to the minimum value sufficient to maintain the contact, and then the tip was moved to a new position at least $40 \mathrm{~nm}$ away, where a desired force load was applied and the bias scan was repeated. In the force region between 4.4 and $46.2 \mathrm{nN}$, stable and reproducible $I-V$ relations were observed. Under these conditions, the tip was expected to be on the monolayer surface rather than to penetrate the monolayer, because the CSAFM tip radius ( $<35 \mathrm{~nm}$ as given in the manual) was much larger than the sectional area of an alkyl molecule $\left(\sim 0.24 \mathrm{~nm}^{2}\right.$ for alkanethiol SAMs on gold $\left.^{46}\right)$. This has also been proved by high-resolution AFM observation since no obvious change of the monolayer topography was observed after $I-V$ measurements in this force region. Figure 6 a clearly shows that the $I-V$ curves shifted positively as force was increased. The force-dependent $I-V$
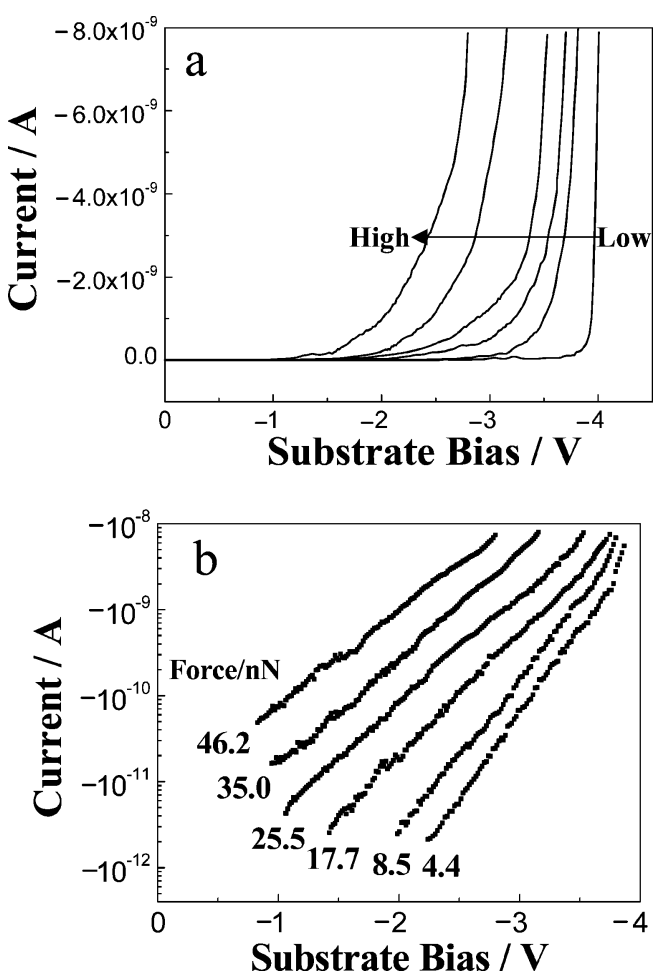

Figure 6. $I-V$ curves obtained at various forces for the $\mathrm{C} 12$ monolayer on $\mathrm{n}-\mathrm{Si}(111)$. The current is given in (a) linear coordinates and (b) log coordinates.

curve shifts have also been found for alkanethiol SAMs on gold by using interfacial force microscopy ${ }^{47}$ and current sensing atomic force microscopy. ${ }^{31}$ The only difference is the shape of the $I-V$ curve, which reflects a difference in electron-transfer mechanisms.

Semilogarithmic $I-V$ relations are presented in Figure $6 \mathrm{~b}$, which shows $\log (i)$ is proportional to the bias even though the slopes are slightly different. At a given bias, $-2.5 \mathrm{~V}$ for example, the current increases drastically with increase in force, as shown by the squares in Figure 7. Two main factors may contribute to the stress-induced current increase. First, when the CSAFM tip pushes the soft monolayer, the monolayer is correspondingly deformed, leading to a larger contact area. The contact area can be roughly estimated from the Hertzian model, ${ }^{48}$ which describes a spherical tip in contact with an elastic halfspace. However, the Hertzian model neglects the effect from interfacial adhesion, which could be important at relatively small 


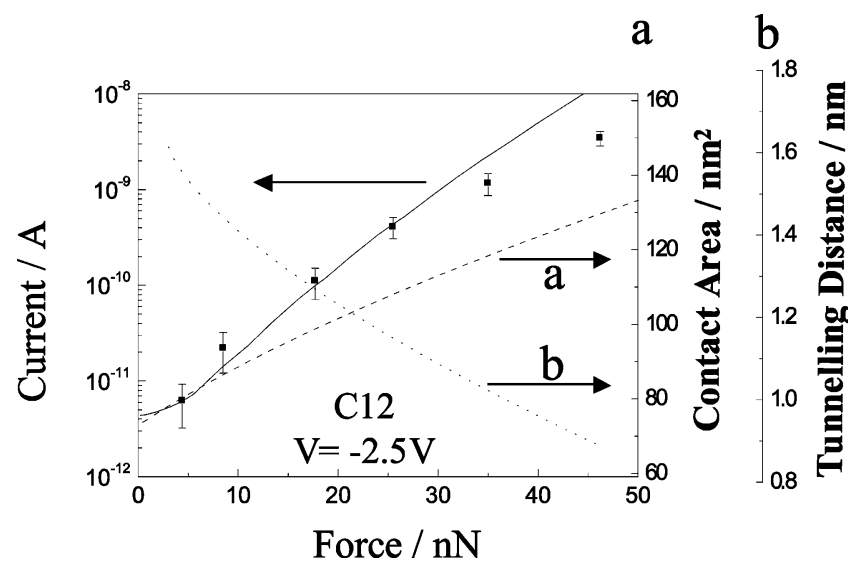

Figure 7. Force dependence of the current obtained at $-2.5 \mathrm{~V}$ in Figure 6 . The squares and solid line show experimental data and the simulated result, respectively. The force dependencies of the contact area (dashed line) and tunneling distance (dotted line) are also shown.

stress. The Johnson-Kendall-Roberts (JKR) model ${ }^{49}$ takes into account this effect and is appropriate for predicting the contact area. According to the JKR model, the radius of the contact area, $A$, can be expressed by

$$
A^{3}=\frac{3 R}{4} \frac{1-v^{2}}{E}\left[F+3 F_{0}+2 \sqrt{F_{0} F+\left(F_{0}\right)^{2}}\right]
$$

where $v$ is the Poisson number, which is 0.3 for most materials, $R$ is the radius of the CSAFM tip, which is $<35 \mathrm{~nm}$ according to the manufacturer and is assumed to be $30 \mathrm{~nm}$ in this study, $E$ is the elastic modulus, which is $1.0 \times 10^{10} \mathrm{~N} \mathrm{~m}^{-2} 50$ for the alkyl monolayers, and $F_{0}$ is the adhesive force, which is 11.0 $\pm 1.9 \mathrm{nN}$ as described before. The evolution of the contact area with the applied force is given by the dashed line in Figure 7.

Second, the deformation of SAMs under stress may result in a shortened tunneling distance, which may affect the tunneling current according to the exponential tunneling eq $2 .{ }^{10,12,31,32}$ The shortest tunneling distance under stress can be simply treated by the Hertzian model ${ }^{48}$ as

$$
\left(d_{0}-d\right)^{3}=\frac{9}{16}\left(\frac{1-v^{2}}{E}\right)^{2} \frac{F^{2}}{R}
$$

where $d$ and $d_{0}$ are the tunneling distance with and without stress, respectively. ${ }^{51}$ The evolution of the tunneling distance is shown by the dotted line in Figure 7. Generally, when the electron flows through a section, the current is proportional to the sectional area. This relation is also consistent with the experimental data in determination of molecular resistance. ${ }^{11}$ Thus, the total tunneling current measured by the CSAFM can be expressed as the sum of the current through each contact molecule.

$$
i=\sum_{j} i_{0} \exp \left(-\beta d_{j}\right)
$$

where $i_{0}$ is the current through alkyl molecules at distance $d_{0}$. $d_{j}$ represents the compressed vertical distance for each molecule linking the tip and substrate. The minimum value of $d_{j}$ was obtained from eq 8 , and the other values were determined by considering the semispherical tip shape with a radius of $30 \mathrm{~nm}$. The number of molecules in contact was calculated by using eq 7 with the molecular area. ${ }^{46}$ The current-applied force relation was simulated by eq 9 and is shown by a solid line in Figure 7. This relation is in good agreement with the experi-

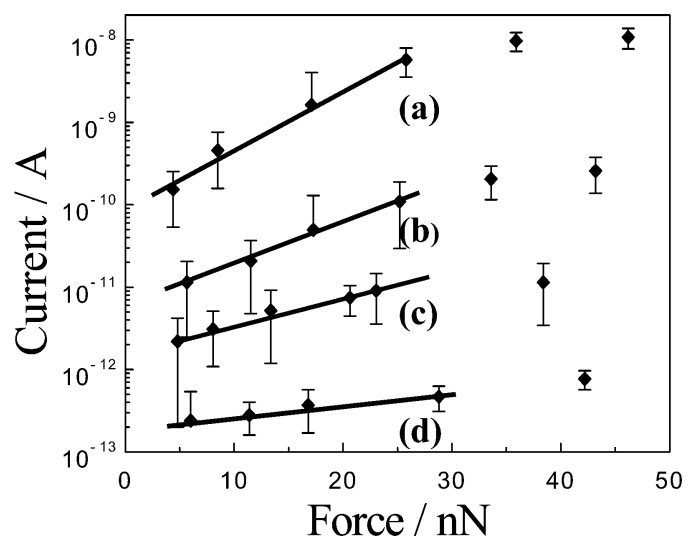

Figure 8. Force dependence of the current obtained at $-3.5 \mathrm{~V}$ for curves for (a) C12, (b) C14, (c) C16, and (d) C18 monolayers.

mental data. One must note that while the contact area was increased by ca. 5 times, the current was increased by almost 3 orders of magnitude as the force was changed from 4.4 to 46.2 $\mathrm{nN}$. This clearly shows that the variation of the tunneling distance under stress is the dominant factor for the increase in the current. Although the monolayer deformation model may simulate accurately the current increase under stress, it cannot give any insight into the variation of the slopes of the $\log (i)-V$ relation shown in Figure 5b. Therefore, other force-induced electron-transfer mechanisms, including the force-induced shift of the highest occupied molecular orbital (HOMO) and the lowest unoccupied molecular orbital (LUMO) of the monolayers $^{47}$ and the contribution of hole transport, ${ }^{9,13}$ must be involved in this process.

Although the force dependence of the current is shown in Figure 7 for the $\mathrm{C} 12$ monolayer on $\mathrm{Si}(111)$ at a given bias of $-2.5 \mathrm{~V}$, this bias is not very appropriate to compare the force dependencies of the current at a constant bias for all four samples. As shown in Figure 3b, the longer the alkyl chain, the more negative the shift in the $I-V$ curve. To evaluate the forcedependent current response for all samples, the bias at which extrapolation of the $\log (i)$ is as small as possible must be selected. A suitable value would be $-3.5 \mathrm{~V}$. Figure 8 shows four sets of current response to force stress at $-3.5 \mathrm{~V}$, where $\log (i)$ can be roughly treated as a linear increase in the force region between 5 and $30 \mathrm{nN}$. These results imply that the deformation of an alkyl monolayer in a moderate force region is elastic. This agrees with observations using an interfacialforce microscope. ${ }^{52}$ The sensitivity of the monolayer structure to the stress can be roughly represented by the slopes in Figure 8. The slope decreases with an increase in chain length, indicating that the monolayer of longer chain molecules is more robust. This observation agrees well with the results of AFM friction measurements ${ }^{53}$ and with the theoretical prediction that shows the stabilization energy of an alkyl monolayer is proportional to the number of $\mathrm{CH}_{2}$ units. ${ }^{54}$

\section{Conclusions}

Alkyl monolayers were fabricated directly on a H-terminated $\mathrm{Si}(111)$ by thermal addition of alkenes and were investigated in detail by using a CSAFM. The use of a semiconductor electrode in this junction leads to a different electron tunneling mechanism as compared to the junction with a metal electrode. Free electrons at the interface are thought to be in a thermal equilibrium with electrons in the bulk and to tunnel through the alkyl monolayers to the metal electrode. The blockage of electron transfer between the silicon electrode and the Pt-coated AFM tip can be modulated by controlling the monolayer 
thickness, that is, the length of the alkyl chain. Further study on the chain length dependence of the tunneling current gave a tunneling coefficient, $\beta$, which was dependent on bias as predicted by the Simmons electron tunneling model. The $I-V$ relation was found to be sensitive to the applied force, reflecting the fact that the organic monolayer is a soft material. Contact area and tunneling distance were considered to be the main reason for the force dependence of $I-V$ curves.

An organic insulating monolayer fabricated directly onto a semiconductor surface has many advantages, for example, a clear interface structure, high packing density, controllable insulator thickness, and high dielectric strength as well as being easy to fabricate and further functionalize; it is therefore a very promising component for future electronic devices.

Acknowledgment. This work was partially supported by a Grant-in-Aid for Scientific Research from the Ministry of Education, Culture, Sports, Science and Technology, Japan. J.Z. is supported by a Monbukagakusho Scholarship.

\section{References and Notes}

(1) Wayner, D. D. M.; Wolkow, R. A. J. Chem. Soc., Perkin Trans. 2002, 2, 23 .

(2) Wagner, P.; Nock, S.; Spudich, J. A.; Volkmuth, W. D.; Chu, S.; Cicero, R. L.; Wade, C. P.; Linford, M. R.; Chidsey, C. E. D. J. Struct. Biol. 1997, 119, 198.

(3) Strother, T.; Hamers, R. J.; Smith, L. M. Nucleic Acids Res. 2000, 28,3535 .

(4) Yates, J. T., Jr. Science 1998, 279, 335.

(5) Boulas, C.; Davidovits, J. V.; Rondelez, F.; Vuillaume, D. Phys. Rev. Lett. 1996, 76, 4797.

(6) Vuillaume, D.; Boulas, C.; Collet, J.; Davidovits, J. V.; Rondelez, F. Appl. Phys. Lett. 1996, 69, 1646.

(7) Gurtner, C.; Wun, A. W.; Sailor, M. J. Angew. Chem., Int. Ed. 1999, $38,1966$.

(8) Bansal, A.; Lewis, N. S. J. Phys. Chem. B 1998, 102, 1067. 2886.

(9) Selzer, Y.; Salomon, A.; Cahen, D. J. Am. Chem. Soc. 2002, 124,

(10) Cui, X. D.; Zarate, X.; Tomfohr, J.; Sankey, O. F.; Primak, A.; Moore, A. L.; Moore, T. A.; Gust, D.; Harris, G.; Lindsay, S. M. Nanotechnology 2002, 13, 5 .

(11) Cui, X. D.; Primak, A.; Zarate, X.; Tomfohr, J.; Sankey, O. F.; Moore, A. L.; Moore, T. A.; Gust, D.; Harris, G.; Lindsay, S. M. Science 2001, 294, 571.

(12) Wold, D. J.; Frisbie, C. D. J. Am. Chem. Soc. 2001, 123, 5549.

(13) Beebe, J. M.; Engelkes, V. B.; Miller, L. L.; Frisbie, C. D. J. Am. Chem. Soc. 2002, 124, 11268.

(14) Linford, M. R.; Chidsey, C. E. D. J. Am. Chem. Soc. 1993, 115, 12631 .

(15) Linford, M. R.; Fenter, P.; Eisenberger, P. M.; Chidsey, C. E. D. J. Am. Chem. Soc. 1995, 117, 3145.

(16) Boukherrroub, R.; Morin, S.; Bensebaa, F.; Wayner, D. D. M. Langmuir 1999, 15, 3831 .

(17) Boukherroub, R.; Wayner, D. D. M. J. Am. Chem. Soc. 1999, 121, 11513

(18) Yu, H.; Morin, S.; Wayner, D. M.; Allongue, P.; Henry de Villeneuve, C. H. J. Phys. Chem. B 2000, 104, 11157.

(19) Sieval, A. B.; Vleeming, V.; Zuilhof, H.; Sudholter, E. J. R. Langmuir 1999, 15, 8288.
(20) Terry, J.; Linford, M. R.; Wigren, C.; Cao, R.; Pianetta, P.; Chidsey, C. E. D. Appl. Phys. Lett. 1997, 71, 1056

(21) Cheng, J.; Robinson, D. B.; Cicero, R. L.; Eberspacher, T.; Barrelet, C. J.; Chidsey, C. E. D. J. Phys. Chem. B 2001, 105, 10900.

(22) Effenberger, F.; Gotz, G.; Bidlingmaier, B.; Wezstein, M. Angew. Chem., Int. Ed. 1998, 37, 2462.

(23) Henry de Villeneuve, C.; Pinson, J.; Bernard, M. C.; Allongue, P. J. Phys. Chem. B 1997, 101, 2415.

(24) Fidelis, A.; Ozanam, F.; Chazalviel, J. N. Surf. Sci. 2000, 444, L7.

(25) Bansal, A.; Li, X.; Lauermann, I.; Lewis, N. S. J. Am. Chem. Soc. 1996, 118,7225

(26) Bansal, A.; Lewis, N. S. J. Phys. Chem. B 1998, 102, 4058.

(27) Liu, J.; Yu, H. J. Phys. Chem. B 2003, 107, 7803.

(28) Liu, J.; Yu, H. ChemPhysChem 2003, 4, 335.

(29) Liu, J.; Waugh, D. M.; Yu, H. Appl. Phys. Lett. 2002, 81, 4967.

(30) Zhao, J.; Uosaki, K. Langmuir 2001, 17, 7784.

(31) Zhao, J.; Uosaki, K. Nano Lett. 2002, 2, 137.

(32) Wold, D. J.; Frisbie, C. D. J. Am. Chem. Soc. 2000, 122, 2970.

(33) Quayum, M. E.; Kondo, T.; Nihonyanagi, S.; Miyamoto, D.; Uosaki, K. Chem. Lett. 2002, 2, 208.

(34) Nihonyanagi, S.; Miyamoto, D.; Idojiri, S.; Uosaki, K. J. Am. Chem. Soc. 2004, 126, 7034 .

(35) Holmlin, R. E.; Haag, R.; Chabinyc, M. L.; Ismagilov, R. F.; Cohen, A. E.; Terfort, A.; Rampi, M. A.; Whitesides, G. M. J. Am. Chem. Soc. 2001, 123, 5075 .

(36) Zhao, J.; Uosaki, K. Appl. Phys. Lett. 2003, 83, 2034.

(37) Sugimura, H.; Nakagiri, N. Langmuir 1995, 11, 3623.

(38) Sugimura, H.; Takai, O.; Nakagiri, N. J. Electroanal. Chem. 1999, $473,230$.

(39) Card, H. C.; Rhoderick, E. H. J. Phys. D: Appl. Phys. 1971, 4, 1589 .

(40) Simmons, J. G. J. Appl. Phys. 1963, 34, 1793.

(41) Likharev, K. K. Proc. IEEE 1999, 87, 606.

(42) Rhoderick, E. H.; Williams, R. H. Metal-Semiconductor Contacts, 2nd ed.; Clarendon Press: Oxford, 1988.

(43) Soliman, M. M. Renewable Energy 2001, 23, 483.

(44) Nuhoglu, C.; Ayyildiz, E.; Saglam, M.; Turut, A. Appl. Surf. Sci. 1998, 135, 350 .

(45) Liu, G. Y.; Xu, S.; Qian, Y. Acc. Chem. Res. 2000, 33, 457.

(46) Nelles, G.; Schonherr, H.; Jaschke, M.; Wolf, H.; Schaub, M.; Kuther, J.; Tremel, W.; Bamberg, E.; Ringsdorf, H.; Butt, H. J. Langmuir 1998, 14, 808 .

(47) Son, K. A.; Kim, H. I.; Houston, J. E. Phys. Rev. Lett. 2001, 86, 5357.

(48) Timoshenko, S. P.; Goodier, J. N. Theory of Elasticity; McGrawHill: New York, 1970 .

(49) Johnson, K. L.; Kendall, K.; Roberts, A. D. Proc. R. Soc. London, Ser. A 1971, 324, 301.

(50) Weihs, T. P.; Nawaz, Z.; Jarvis, S. P.; Pethica, J. B. Appl. Phys. Lett. 1991, 59, 3536.

(51) Not the JKR model but the Hertzian model was used to estimate the force-dependent tunneling distance because the simulation based on the latter is much easier. Although the former gives more accurate values, particularly in the adhesive force region, the difference becomes less and less as the tip stress increases and essentially no difference is expected for the present condition of very large stress.

(52) Joyce, S. A.; Thomas, R. C.; Houston, J. E.; Michalske, T. A.; Crooks, R. M. Phys. Rev. Lett. 1992, 68, 2790.

(53) Xiao, X.; Hu, J.; Charych, D. H.; Salmeron, M. Langmuir 1996, 12,235 .

(54) Israelachvili, J. Intermolecular and Surface Forces, 2nd ed.; Academic Press: New York, 1992. 